Baltic Astronomy, vol.1, 59-81, 1992.

\title{
SOME OBSERVABLE CHARACTERISTICS OF SYMBIOTIC STARS FROM MODEL CALCULATIONS
}

\author{
Laurits Leedjärv \\ Tartu Astrophysical Observatory, \\ Töravere 202444, Estonia
}

Received May 31, 1991.

\begin{abstract}
Energy distributions in continuous spectra of various models of symbiotic stars in the wavelength range from $25 \AA$ to $20 \mathrm{~cm}$ have been computed. The impact of different input parameters on the shape of the continuum is investigated. It has been found that for models with a blackbody as the hot component of the symbiotic system both the effective temperature and the radius of the blackbody are very sensitive parameters, affecting remarkably the shape of the continuum. In the case of an accretion disk, the radius of the accreting star and the accretion rate are the most sensitive parameters while the mass of the accretor and the inclination angle of the disk affect the computed colour indices only a little. The influence of ionized gas and a cold component is studied as well. Location of the computed models on two-colour diagrams $U-B, B-V$ and in the far-infrared region $(12-100 \mu \mathrm{m})$ is investigated. It has been found that on the far-infrared diagrams S- and D-type symbiotic stars can be easily distinguished.
\end{abstract}

Key words: binary stars - symbiotic stars - stellar models

\section{Introduction}

Symbiotic stars have been of great interest to astronomers since the 1930s. Nowadays it is generally accepted that symbiotic stars are interacting binaries consisting of a red giant and a hot dwarf star which ionizes the red giant's wind. In our previous papers (Leedjärv, $1989,1990)$ physical characteristics of 15 symbiotic stars have been 
investigated. We have applied the technique of comparing the computed energy distribution in the continuous spectrum with the observed one. The computed energy distributions may be of interest for certain theoretical purposes. In the following we shall investigate how different values of various parameters of a symbiotic system change the shape of the computed continuous spectrum, as a result of which some observable characteristics of the star, e.g. colour indices change, too. Some conclusions and possible implications will be brought out.

\section{Methods}

Models of symbiotic stars and methods for computing their continuous spectra have been described in detail in our previous paper (Leedjärv, 1988). Here we only outline some basic concepts. As a rule, a model of a symbiotic star includes four components: (1) a red giant of the spectral class $\mathrm{K}$ or $\mathrm{M}$, in some cases a Mira variable; (2) a hot companion - a subdwarf star without accretion, or an accretion disk around a white dwarf, a subdwarf or a main-sequence star; (3) a gaseous envelope consisting of ionized hydrogen and doubly ionized helium, the size of the ionized volume being determined by the luminosity of the hot component; (4) an envelope of cold dust around the giant star (present not in all cases).

The continuous spectrum of the red giant has been taken according to the papers by Straižys and Sviderskiene (1972) and Sviderskiene (1974), i.e the averaged observed snergy distributions for stars of various spectral and luminosity classes. In the case of the hot component the blackbody approximation is used for the subdwarf star, while the energy distribution in the spectrum of the accretion disk is computed following the papers by Shakura and Sunyaev (1973) and Kenyon and Webbink (1984). A part of the stellar wind of the red giant will be ionized by the hot component, and this gas radiates in the continuous spectrum due to free-free and free-bound electron transitions (in some cases hycirogen two-photon emission may also be significant). For determining the volume of the ionized gas we use the concept of the Sirömgren sphere. In the case of dust envelopes (those may exist in about 20-40 per cent of all symbiotic systems) again biackbody approximation is lised.

In the preseni paper continuous specira of symbiotic systems in the wavelength range from $25 \AA$ to $20 \mathrm{~cm}$ are computed. Usu- 
ally 144 points in this range are used, including the most important molecular bands of $\mathrm{TiO}$ and the boundaries of spectral series of hydrogen and helium. Comparing such theoretical continuous spectra with the observed energy distribution in the continuum of individual symbiotic stars we may vary input parameters of computation until the best agreement is achieved, and in this way we get main physical characteristics of the symbiotic star with certain accuracy. Such an approach has been used by us in the papers Leedjärv (1989, 1990 ). When computing theoretical energy distributions, some problems arise, e.g. which are the most sensitive parameters of the symbiotic system affecting most of all the observable characteristics of the star. A study of various theoretical dependencies helps us to determine the parameters of individual stars more unambiguously, and the results presented in the present paper have already been partially used in our previous papers, but here we summarize some of them in a systematic way.

The shape of the continuous spectrum can be characterized by colour indices. We have used the Johnson photometric system with the bands $U B V R I J H K L M N$. Using the filter response functions by Johnson (1965) (for $U, B$ and $V$ modified by Straižys, 1977), the most important colour indices as $U-B, B-V, V-R, J-K$, etc. are computed for various theoretical spectra of symbiotic stars. We have made use also of the IRÁS satellite data in the far-infrared region and the indices $[12]-[25],[25]-[60]$ and $[60]-[100]$ have been computed, taking the response functions by Beichman et al. (1985). It should be mentioned that colour indices obtained this way may not exactly correspond to the observed ones because many physical simplifications have been made in the computations. At the same time, the observed colour indices of most symbiotic stars are variable. However, such theoretical indices are suitable to investigate the dependency of the shape of the continuous spectrum on the values of different parameters. In addition to colour indices, some other characteristics, which can be observed or indirectly estimated, have been computed. Those are, for example, the Balmer jump $D_{\mathrm{B}}$, the mass of the ionized gas $M_{\text {gas }}$ and the observabie radio flux $F_{\nu}$.

\section{Hot component as a blackbody}

One type of models of symbiotic stars contains hot subdwarf stars as hot components. Although model atmosphere fluxes for 
hot stars are available, it has been argued by Kaler (1976) and by Kenyon and Webbink (1984) that for many purposes the blackbody approximation is satisfactory (and in some cases it is even more suitable than model atmospheres). With some restriction we can also use blackbody approximation, if the hot component is a dwarf star accreting matter from the spherically-symmetric stellar wind of the red giant. For the blackbody-type hot component in a symbiotic system, the most significant input parameters in computations of the continuous specirum are the radius and the effective temperature.

\subsection{Radius of the hot component}

The observable flux from a star of a given effective temperature in the blackbody assumption is proportional to $R^{2}$. Let us consider how this radius affects the spectrum of the whole symbiotic system. Figs. 1 and 2 represent some computed dependencies for effective temperatures of the hot component $75000 \mathrm{~K}$ and $40000 \mathrm{~K}$, respectively. The most striking feature in both Figures is the strong dependence of the index $U-B$ on the radius of the hot component, while the index $B-V$ changes rather moderately. Such a behaviour of colour indices is understandable, if we consider contributions which the radiation by the ionized gas makes up from the total energy output of the symbiotic system. In the ultraviolet region (at $1600 \AA$ and $2400 \AA$ ) this fraction does not depend on the radius of the hot component, while at $3200 \AA$ the dependence is remarkable: from 0.48 at $R=0.02 R_{\odot}$ to 0.89 at $R=0.3 R_{\odot}$ for the model with $T_{\text {eff }}=$ $75000 \mathrm{~K}$ and from 0.20 at $R=0.05 R_{\odot}$ to 0.52 at $R=0.8 R_{\odot}$ for the model with $T_{\text {eff }}=40000 \mathrm{~K}$. At longer wavelengths the contribution of the ionized gas again becomes less significant. So, the rapid change of the index $U-B$ in Figs. 1 and 2 is connected with the increase in the volume of the ionized gaseous envelope surrounding the hot component (compare with the curve $M_{\text {gas }}$ in Figs. 1 and 2) and with the fact that in the $U$ bandpass the ionized gas yields a significant portion from the radiation of the whole system. In this context it is not surprising that colours in the far-infrared region remarkably change with the radius of the hot component. In a wavelength region $\lambda \geq 25 \mu \mathrm{m}$ free-free electron transitions in the ionized gas yield a significant part of the radiation of the symbiotic system, and thus, the radius of the hot component affects the outcoming spectrum through the number of ionizing photons. 
Fig. 1. Dependence of colour indices, the Balmer jump -0.8 $D_{B}$ and the mass of ionized gas $M_{\text {gas }}$ on radius of the hot component. Other parameters of the symbiotic system are: for the cold component $\mathrm{Sp}=$ M3III, $T_{\text {eff }}=$ $3600 \mathrm{~K}, R=100 R_{\odot \odot}$; for the ionized gas $T_{e}=10000 \mathrm{~K}$, $n_{e}=n_{\mathrm{HI}}=10 n_{\mathrm{HeII}}=$ $1.0 \cdot 10^{7} \mathrm{~cm}^{-3}$ and for the hot component $T_{\text {hot }}=75000 \mathrm{~K}$.

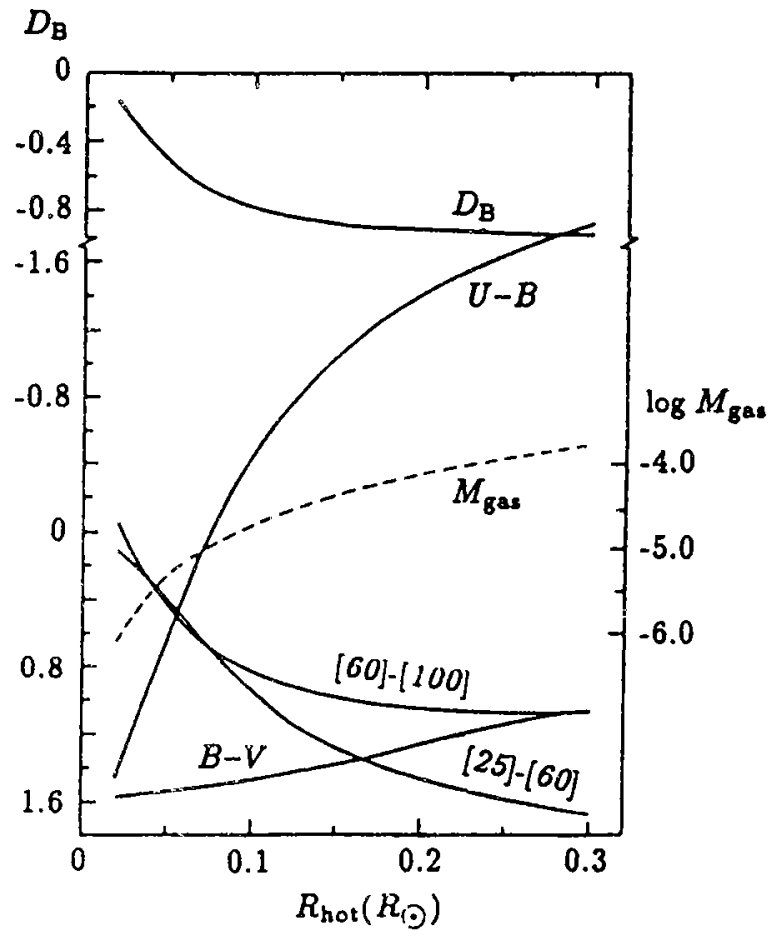

Fig. 2. The same as in Fig. 1, with temperature of the hot component $T_{\text {hot }}=40000 \mathrm{~K}$.

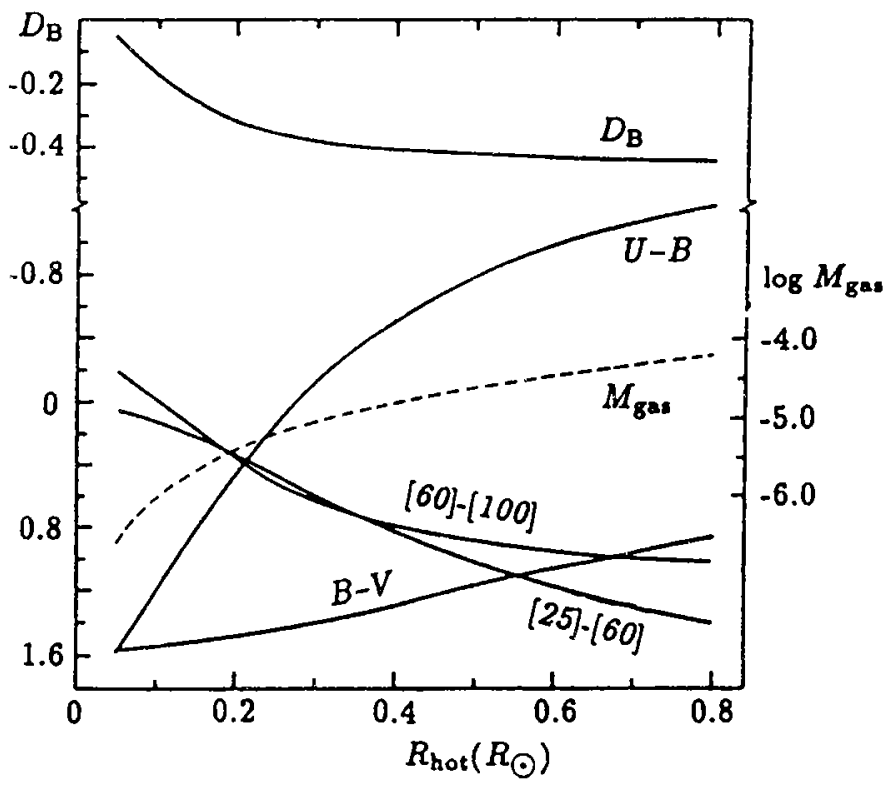




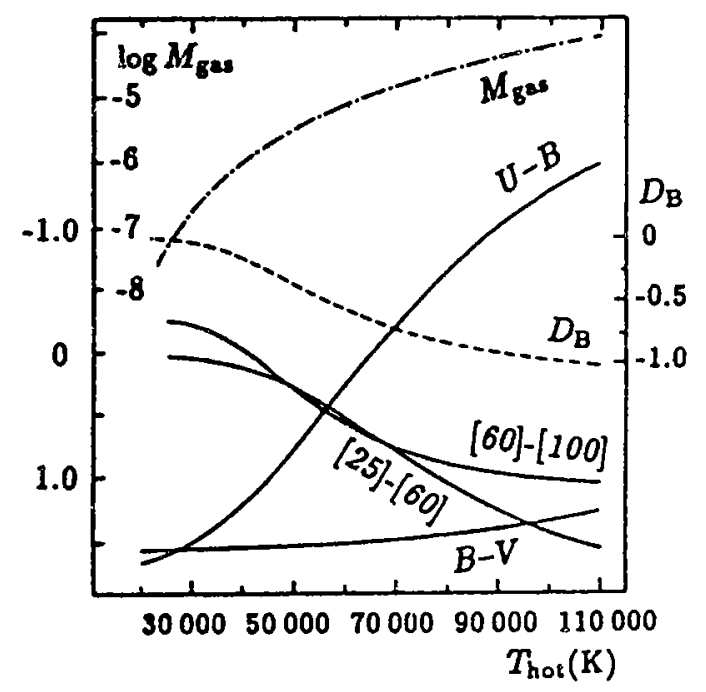

Fig. 3. Dependence of colour indices, the Balmer jump $D_{\mathrm{B}}$ and the mass of ionized gas $M_{\text {gas }}$ on temperature of the hot component. The cold component and ionized gas are as in Figs. 1 and 2, the radius of the hot component $R_{\text {hot }}=$ $0.10 R_{\odot}$.

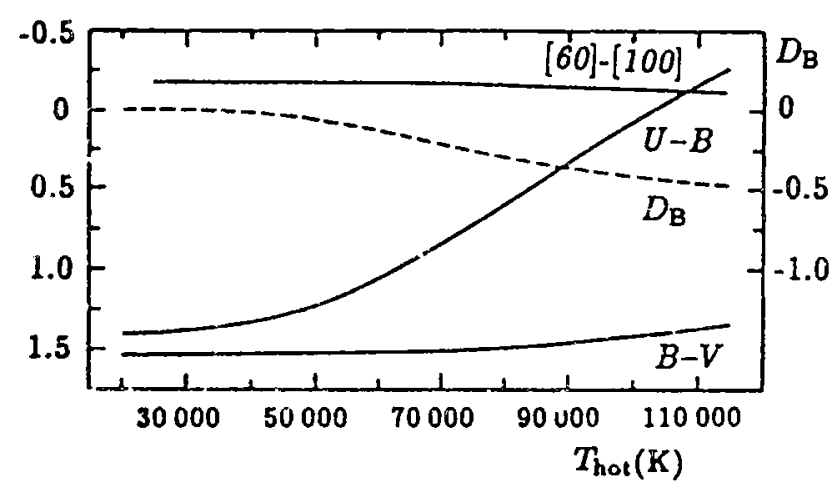

Fig. 4. Dependence of colour indices and the Balmer jump $D_{\mathrm{B}}$ on temperature of the hot component. Other parameters of the symbiotic system are: for the coid component $\mathrm{Sp}=\mathrm{M} 5 \mathrm{III}, T_{\text {eff }}=3300 \mathrm{~K}, R=$ $209 R_{\odot} ;$ for the ionized gas $T_{e}=20000 \mathrm{~K}, n_{\mathrm{c}}=n_{\mathrm{HI}}=10 n_{\mathrm{HeII}}=$ $1.0 \cdot 10^{8} \mathrm{~cm}^{-3}$ and for the dust envelope $T=800 \mathrm{~K}, R=2.0 \cdot 10^{14} \mathrm{~cm}$. 


\subsection{Temperature of the hot component}

The outcoming flux from the blackbody is proportional to $\exp (-h c / \lambda k T)$. Fig. 3 looks quite similar to Figs. 1 and 2. It can be said that the effective temperature of the hot component affects the continuous spectrum of the symbiotic star first of all through the rate at which the hot component can ionize the surrounding gas. This results in a strong dependence of indices $U-B,[25]-[60]$ and $[60]-[100]$ on the effective temperature, while the index $B-V$ changes only a little.

Fig. 4 represents the model with a dust envelope around the red giant. At the temperature $800 \mathrm{~K}$ the maximum radiation of the dust is at about $3.5 \mu \mathrm{m}$, however, the total radiation of the dust and of the red giant dominates over radiation due to free-iree electron transitions also at longer wavelengths. As a result, the index [60][100] depends slightly on the temperature of the hot component (this holds true for the index $[25]-[60]$ as well).

\subsection{Parameters of ionized gas}

As a rule, we consider gas with the density of hydrogen ions $n_{\mathrm{HI}}$ equal to the electron density $n_{e}$, and the density of helium ions $n_{\text {HeII }}$ equal to $0.1 n_{e}$. Thus, the main parameters characterizing ionized gas are the electron density $n_{e}$ and the electron temperature $T_{e}$. The range of possible electron temperatures for symbiotic stars is not wide : from $5000 \mathrm{~K}$ to about $20000 \mathrm{~K}$. Fig 5 shows that there is but weak dependence of computed colour indices and the Balmer jump on the electron temperature in this range. In Fig. 5 the contributions of radiation generated by the ionized gas from the total radiation of the system at different wavelengths are shown as well. Although its dependence on electron temperature is not essential, however, the shape of the Balmer continuum is defined by $T_{e}$.

The electron density $n_{e}$ does not directly modify the shape of the continuous spectrum, but there exists a linear dependence of $\log M_{\text {gas }}$ on $\log n_{e}$. This reflects the fact that gaseous envelopes in symbiotic systems are bound by ionization: the higher the electron density, the smaller the volume ionized by the hot component. Therefore, the volume emission measure $n_{e}^{2} V$ remains constant for all values of $n_{e}$. A similar correlation between $M_{\text {gas }}$ and $n_{e}$ has been obtained studying the models of 15 real symbiotic stars (Leedjärv, 1990) and the same dependence is observed for planetary nebulae. 


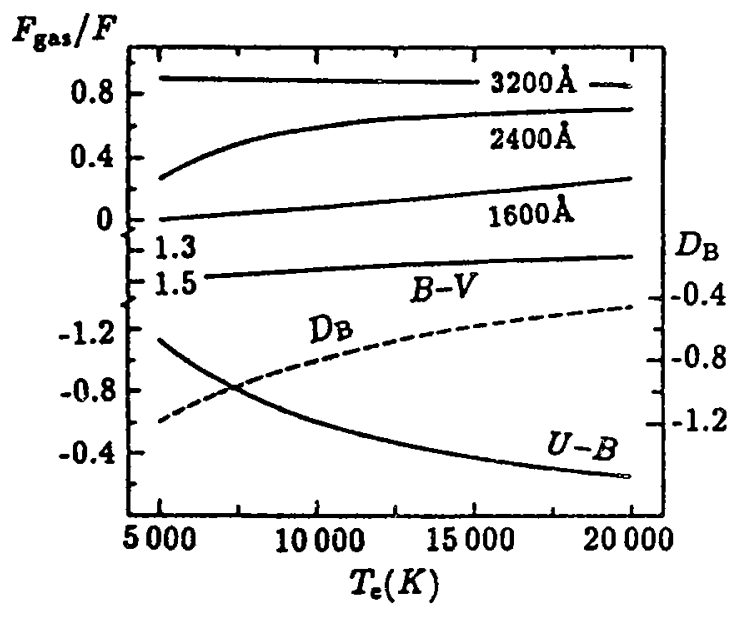

Fig. 5. Dependence of colour indices, the Balmer jump $D_{B}$ and contributions, which the ionized gas makes up from total radiation of the symbiotic system, on the electron temperature. Other parameters are: for the cold component $\mathrm{Sp}=\mathrm{M} 5 \mathrm{III}$, $T_{\text {eff }}=3300 \mathrm{~K}, R=209 R_{\odot}$; for the hot component $T_{\text {hot }}=$ $80000 \mathrm{~K}, R_{\text {hot }}=0.12 R_{\odot}$ and for the ionized gas $n_{e}=$ $n_{\mathrm{HI}}=10 n_{\mathrm{HeII}}=1.0 \cdot 10^{7}$ $\mathrm{cm}^{-3}$.

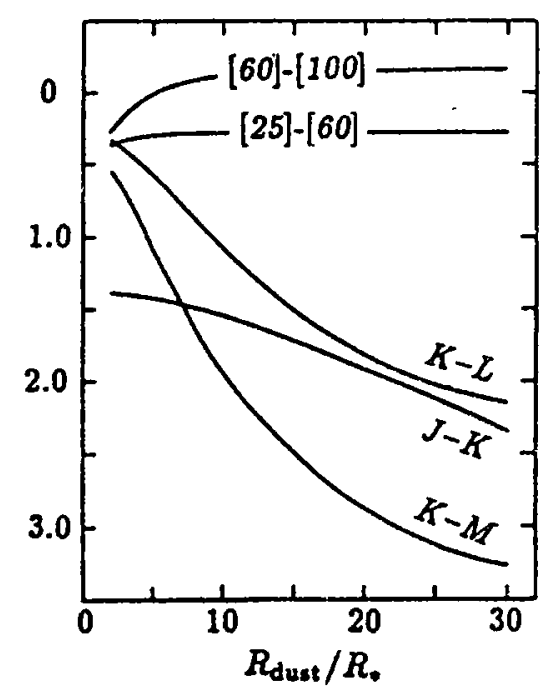

Fig. 6. Dependence of infrared colour indices on radius of the dust envelope $\left(R_{*}\right.$ is radius of the red giant) with the temperature $T=800 \mathrm{~K}$. Other parameters of the symbiotic system are: for the cold component $\mathrm{Sp}=\mathrm{M} 6 \mathrm{III}, T_{\text {eff }}=3200 \mathrm{~K}, R=$ $295 R_{\odot} ;$ for the hot component $T_{\text {hot }}=80000 \mathrm{~K}, R_{\text {hot }}=0.12 R_{\odot}$ and for the ionized gas $T_{e}=$ $10000 \mathrm{~K}, n_{e}=n_{\mathrm{HI}}=10 n_{\mathrm{HeII}}=$ $1.0 \cdot 10^{7} \mathrm{~cm}^{-3}$. 


\subsection{Dust envelopes}

In some symbiotic stars the red giant component is surrounded by a dust envelope. Usually, the temperature of the dust is found to be about $800-1000 \mathrm{~K}$, but as it has been shown by Kenyon, Fernandez-Castro and Stencel (1988), the data by IRAS often suggest to prefer values $300-450 \mathrm{~K}$. In some cases, a better agreement between theory and observations is obtained assuming the presence of two dust envelopes with different temperatures (e.g. Anandarao and Pottasch, 1986; Anandarao, Taylor and Pottasch, 1988).

Fig. 6 demonstrates a dependence of some infrared colour indices on the radius of the spherical dust envelope with the temperature $800 \mathrm{~K}$. As expected, the effect is the greatest in the region $2.2-5.0$ $\mu \mathrm{m}$, where the dust in this case shows maximum radiation. Variations of the far-infrared colour indices are insignificant, except a weak increase in the index $[60]-[100]$.

The temperature of the dust envelope reveals itself through the values of wavelengths at which the minimums of certain colour indices fall. In Fig. 7 it is expressively shown how those minimums are shifting with the temperature of the dust. The model in Fig. 8 has two dust envelopes, the inner one with the radius $10 R_{*}$ and the temperature $900 \mathrm{~K}$ and the outer one with the radius $30 R_{*}$. As we can see, the temperature of this outer envelope has only a little effect on far-infrared colours of symbiotic stars (and it is completely unnoticeable in other spectral regions).

\subsection{Models on two-colour dzagrams}

To solve many astrophysical problems, it is useful to investigate the position of a star on two-colour diagrams. Fig. 9a represents the $U-B, B-V$ diagram with an indication of locations of the main sequence stars and normal giants and also of hot blackbodies. Special symbols show the positions of various computed models. As expected, symbiotic stars deviate remarkably from the position of normal stars. Models with less luminous hot components $\left(R_{\mathrm{hot}}=0.02 R_{\odot}\right.$ or $\left.T_{\mathrm{hot}}=20000 \mathrm{~K}\right)$ lie quite near to the sequence of normal M-giants (in the lower right corner of Fig. 9a). By increasing the luminosity of the hot component, the position of symbiotic stars deviates step by step from the location of normal stars and blackbodies upwards and to the right. In Fig. 9b we present the models of 15 real symbiotic stars found by us (Leedjärv, 1989). The lowest 


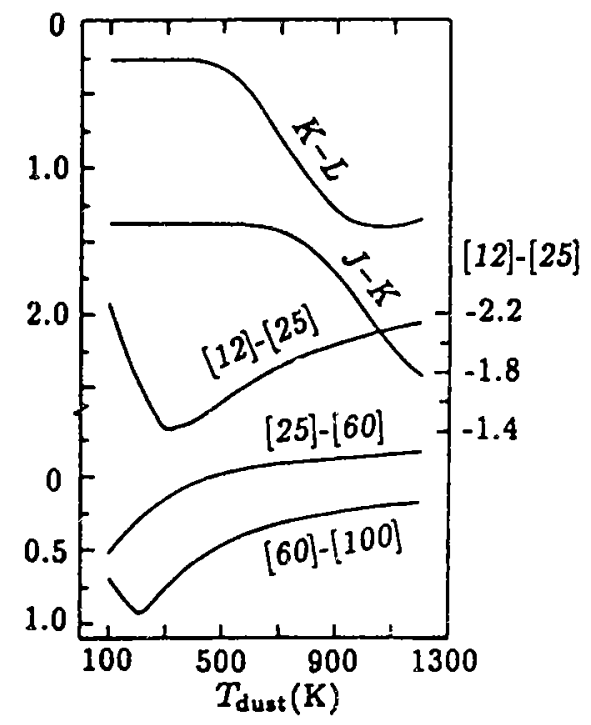

Fig. 7. Dependence of infrared colour indices on temperature of dust envelope with the radius $R=10 R_{*}$. Other parameters of the system are as in Fig. 6.

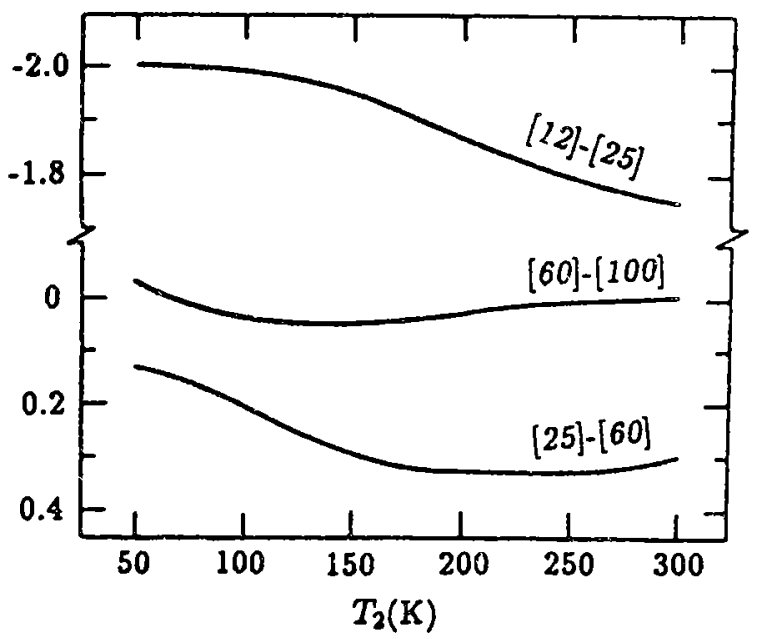

Fig. 8. Dependence of far-infrared colour indices on temperature of the outer dust envelope with the radius $30 R$. For the inner dust envelope $T=900 \mathrm{~K}$ and $R=10 R_{*}$. Other parameters of the system are as in Fig. 6. 
stars in Fig. $9 \mathrm{~b}$ are EG And and R Aqr which, by their optical spectra, are known as low-excitation symbiotic stars. In other words, the position of those stars in the $U-B, B-V$ diagram reflects the fact that the ratio of luminosities $L_{\text {hot }} / L_{\text {cold }}$ is $\leq 0.05$ for those stars, while for other stars it is $\geq 0.1$ and may reach $\sim 4$ for the outburst phase of AG Dra.

In Fig. 9b the models with the hot component as a blackbody and as an accretion disk are noted with different symbols. There are but few points with large scatter and we probably cannot draw any profound conclusion from those data. However, excluding two stars with the brightest accretion disks (RX Pup and BF Cyg), we can at some rate distinguish the models with blackbody-type hot components and the models with accretion disks on the $U-B, B-V$ diagram. However, this conclusion is highly speculative and should be verified by studying the observed colour indices (which, in turn, are highly variable) for a larger number of stars.

For models containing dust envelopes the diagrams with infrared colours can be useful. Using the colours in the near-infrared region $(1.25-5.0 \mu \mathrm{m})$, division of symbiotic stars into types $\mathrm{S}$ (star) and D (dust) has been realized. Lut:d and Tuvikene (1987) investigated the properties of symbiotic stars in the far-infrared (IRAS) region and found that the only useful two-colour diagram is $(K-[12]),([12]-$ [25]) which allows to discriminate between the types S, D and $D^{\prime}$. We tried to make use of colours at 60 and $100 \mu \mathrm{m}$ as well. Fig. 10 represents the diagram $[12]-[25],[25]-[60]$ for models with different dust temperatures and radii.

In Fig. 10 also the positions of computed models for 15 real symbiotic stars from our previous paper (Leedjärv, 1989) are shown. And here we can clearly distinguish D-type (diamonds) and S-type (crosses) symbiotic stars, the latter lying considerably higher than the stars with dust. However, one asterisk (i.e. the model with dust) falls just in the region occupied by S-stars. This point corresponds to the dust temperature $100 \mathrm{~K}$, showing that probably some of symbiotic stars, usually considered as S-stars, actually may contain some amount of cold dust. A similar conclusion was drawn by Luud and Tuvikene (1987). The diagram [25]-[60], [60]-[100] in Fig. 11 is quite similar to the previous one: models with one or two dust envelopes lie almost along the location of blackbodies and that holds true also for real D-type stars. S-type stars again can be quite clearly distinguished from dusty stars. Unfortunately, we have not any $D^{\prime}$-type symbiotic star (cold component of spectral type $\mathrm{G}+$ cold dust) in 


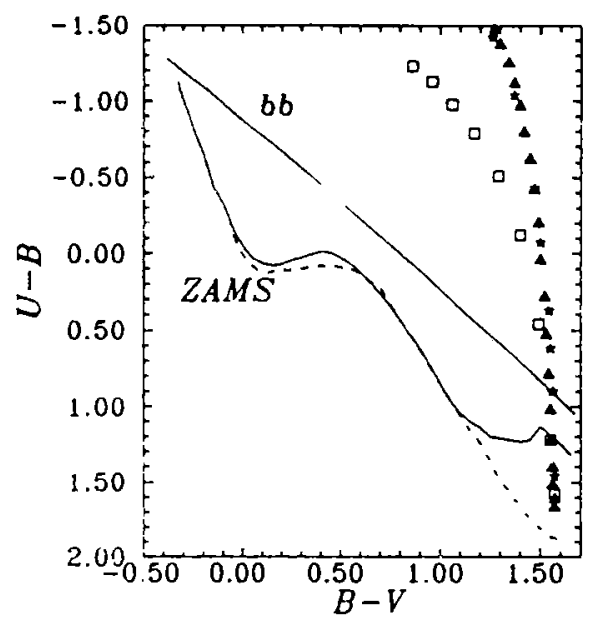

Fig. 8a. Two-colour diagram with locations of the main sequence stars (solid curve marked as ZAMS=Zero-Age Main Sequence), normal red giants (dashed curve) and blackbodies with temperatures from $150000 \mathrm{~K}$ to $3000 \mathrm{~K}$ (solid line). Special symbols mark positions of the computed models of symbiotic stars: pentagons are models from Fig. 1, open squares are models from Fig. 2 and filled triangles are models from Fig. 3.

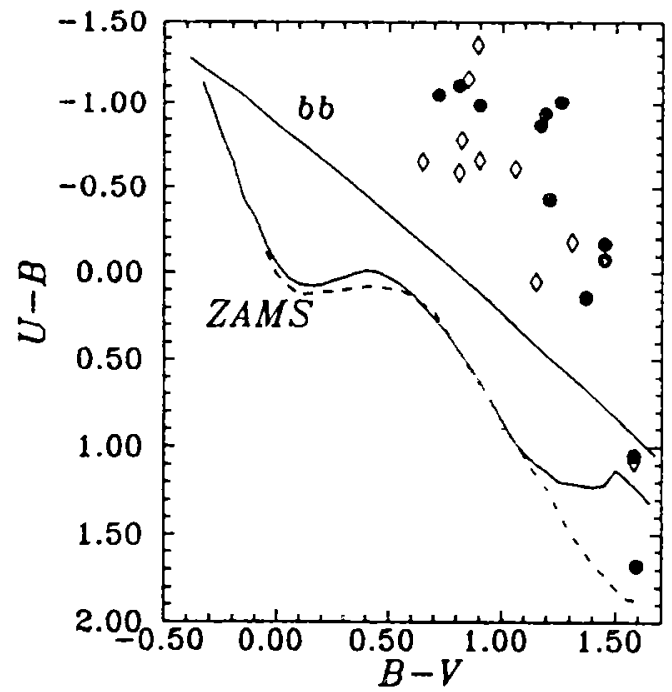

Fig. 9b. Symbols are the same as in Fig. 9a. Special symbols mark the positions of real symbiotic stars by Leedjärv (1989), filled circles mark the hot component as a blackbody and diamonds mark the hot component as an accretion disk. 


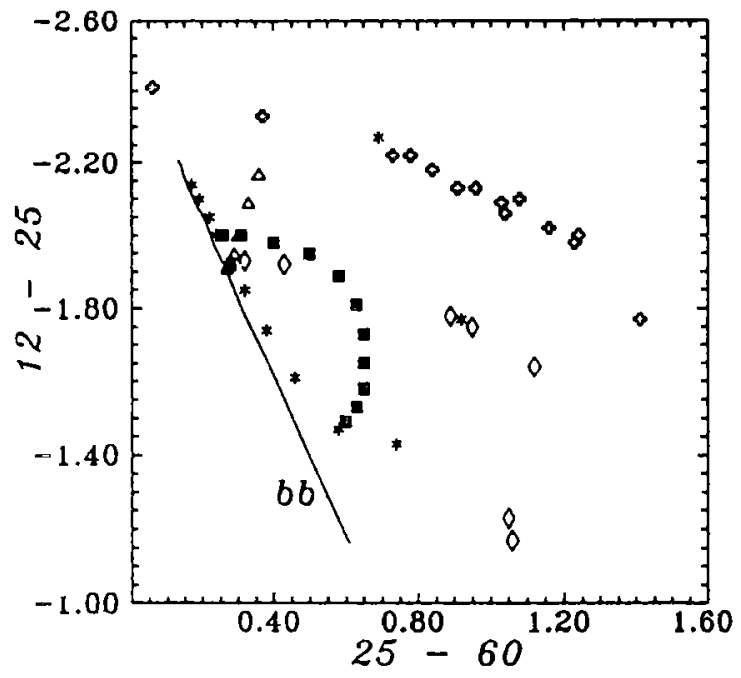

Fig. 10. The [12]-[25], [25]-[60] diagram for models with dust. Besides the location of blackbodies with temperature from $1600 \mathrm{~K}$ to $200 \mathrm{~K}$, open triangles mark models from Fig. 6 (varying radius of the dust envelope), asterisks are models from Fig. 7 (varying temperature of the dust envelope) and filled squares are models from Fig. 8 (varying temperature of the outer dust envelope) Real symbiotic stars by Leedjärv (1989) are denoted by diamonds for type $\mathrm{D}$ (dusty) and crosses for type $\mathrm{S}$ (without dust).

Fig. 11. The $[25]-[60]$, $[60]-[100]$ diagram. Symbols are the same as in Fig. 10. Solid curve marks the location of computed models without dust, with an accretion disk as the hot component, the variable parameter is the accretion rate.

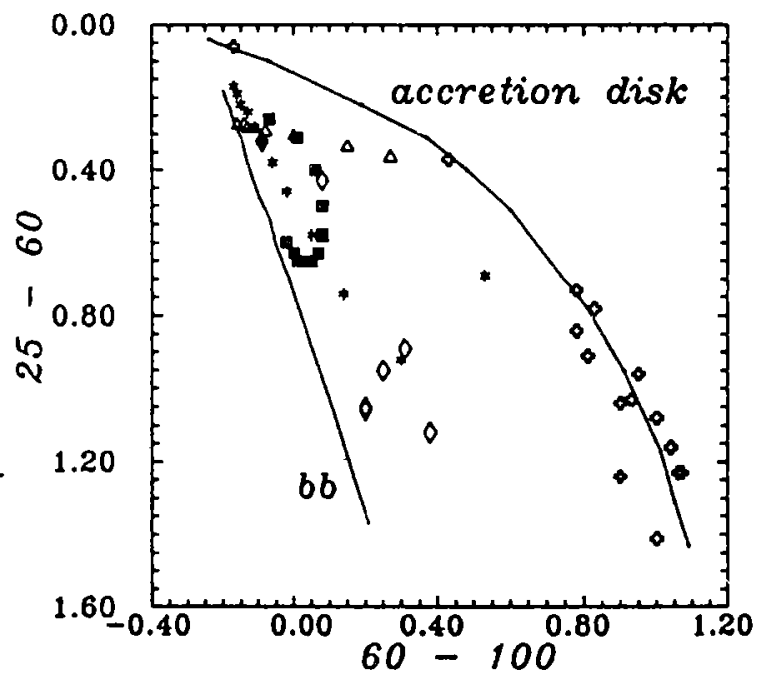




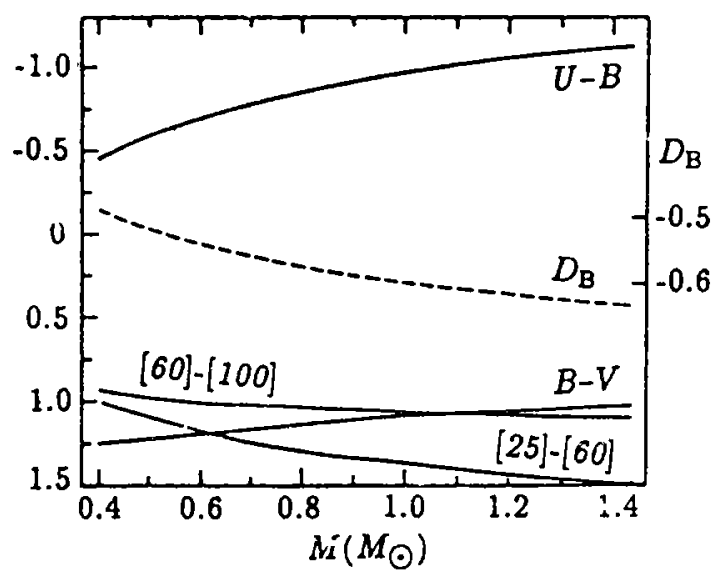

Fig. 12. Dependence of colour indices and the Balmer jump $D_{\mathrm{B}}$ on mass of the accreting star. Other parameters of the symbiotic system are: for the accretor $R_{*}=0.20 R_{\odot}$, $\dot{M}_{\text {acc }}=5.0 \cdot 10^{-7} M_{\odot \mathrm{yr}^{-1}}$, $R_{\text {disk }}=20 R_{*}, i=30^{\circ}$; for the ionized gas $T_{e}=14000 \mathrm{~K}$, $n_{e}=1.0 \cdot 10^{7} \mathrm{~cm}^{-3}$ and for the cold component $\mathrm{Sp}=$ M4III, $T_{\text {eff }}=3500 \mathrm{~K}, R=$ $141 R_{\odot}$

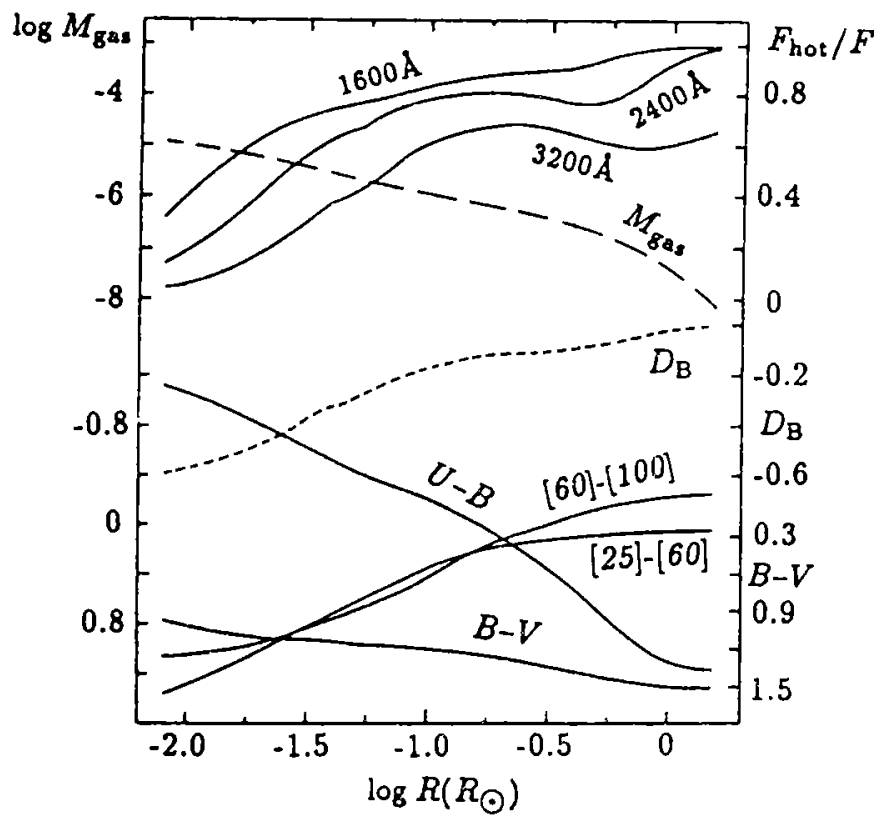

Fig. 13. Dependence of some characteristics on radius of the accreting star. Other parameters of the symbiotic system are: for the accretor $M=0.80 M_{\odot}, \dot{M}_{\text {acc }}=5.0 \cdot 10^{-7} M_{\odot} \mathrm{yr}^{-1}, R_{\text {disk }}=20 R_{*}$ (for a white dwarf), 15R* (for a subdwarf), 10R* (for a main sequence star), $i=30^{\circ}$; for the ionized gas $T_{e}=17000 \mathrm{~K}, n_{e}=1.0 \cdot 10^{8} \mathrm{~cm}^{-3}$ and for the cold component $\mathrm{Sp}=\mathrm{M} 5 \mathrm{III}, T_{\mathrm{eff}}=3300 \mathrm{~K}, R=209 R_{\odot}$. 
our selection, therefore, nothing can be said about the position of real D'-type stars in our two-colour diagrams.

\section{Hot component as an accretion disk}

Interaction of two stars in the form of accretion probably takes place in the majority of symbiotic stars. Different forms of accretion may exist, but in the case of symbiotic stars, besides accretion from spherically-symmetric stellar wind, accretion disks around a white dwarf or a main sequence star are often considered. Below we will consider a steady-state optically thick accretion disk which radiates locally as a blackbody with the temperature depending on the distance from the central star. The boundary layer between the disk and the central star, due to its high temperature $\left(\geq 10^{5} \mathrm{~K}\right)$, also can be considered as a blackbody. When computing the synthetic spectrum of a symbiotic star with an accretion disk, the main free parameters are mass and radius of an accreting star, accretion rate and inclination angle of the disk.

\subsection{Mass and radius of the accretor}

We have computed the values of some colour indices and of the Balmer jump $D_{\mathrm{B}}$ for the range of masses from $0.4 \mathrm{M}_{\odot}$ to $1.4 \mathrm{M}_{\odot}$ (the Chandrasekhar limit for white dwarfs). As one can see from Fig. 12, the dependence of those quantities on mass is not significant. Additional computations show that this holds true also for main sequence accretors in the range of masses $0.5-5.0 \mathrm{M} \odot$. Consequently, when determining the physical parameters of individual symbiotic stars and possessing insufficient information on the properties of an accreting star, we can take its mass as a fixed parameter.

The radius of an accreting star affects the observable characteristics of symbiotic stars in a more remarkable way. As it is known, the less the radius of the accretor, the higher the energy outcome in the accretion phenomenon. From Fig. 13 it is evident that $U-B$ and the far-infrared indices are most sensitive. In other words, the radius of the accretor directly specifies the number of ionizing photons and, accordingly, the amount of radiative ionized gas. This can be well seen in the upper part of Fig. 13.

If the accretor is a white dwarf, its mass and radius are not independent quantities. As the mass of the accretor does not remarkably 
affect the outcoming spectrum, we can find the best fit between the observed and computed energy distributions by varying the radius of the accretor (and other parameters, if necessary). Then the corresponding mass of the accretor can be computed from the mass-radius relation for white dwarfs.

\subsection{Accretion rate and temperature of the boundary layer}

The rate at which the hot component accretes matter from the red giant, is a very important quantity for symbiotic stars. Fig. 14 shows correlation of two colour indices with the accretion rate for a white dwarf accretor. Remarkable changes in the index [60]-[100] can be explained taking into account the fact that the accretion rate is reflected in the emergent spectrum through the amount of the radiative ionized gas, which, in its turn, depends on the accretion luminosity. Fig. 15 shows linear correlation between $\log M_{\text {gas }}$ and $\log \dot{M}$.

In the case of the present model we have also studied radio emission of symbiotic stars. As a rule, it is considered a thermal one, originating from free-free electron transitions in ionized hydrogen (and helium) plasma (e.g. Seaquist and Taylor, 1990). Consequently, we can expect that the radio flux depends on the number of ionizing photons, i.e. on the accretion luminosity. Indeed, as seen from Fig. 15, the radio flux at $4.9 \mathrm{GHz}(6 \mathrm{~cm})$ increases with the accretion rate very steeply for $\dot{M} \geq 10^{-7} M_{\odot} \mathrm{yr}^{-1}$.

Different types of lines in Figs. 14 and 15 mark different methods used for calculating the temperature of the boundary layer. The physics of the boundary layer, a thin region near the accreting star where velocities of accreted particles change from Keplerian values in the disk to zero on the surface of the central star, is quite complicated. Some approximate formulae for the boundary layer temperature are given by Lynden-Bell and Pringle (1974) and Pringle (1977). In the simplest case, if gas pressure and the rotational velocity of the central star can be neglected, the temperature may be approximated by the formula

$$
T_{\mathrm{BL}} \approx 6.5 \cdot 10^{4}\left(\frac{M_{*} \dot{M}}{10^{-5} M_{\odot}^{2} \mathrm{yr}^{-1}}\right)^{1 / 4}\left(\frac{R_{*}}{R_{\odot}}\right)^{-3 / 4}
$$




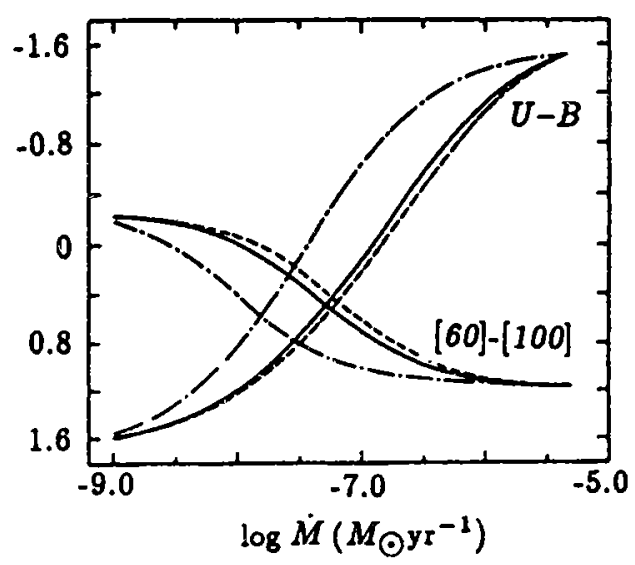

Fig. 14. Dependence of the indices $U-B$ and $[60]-[100]$ on the accretion rate. Other parameters of the symbiotic system are: for the accretor $M=0.80 M_{\odot}, R_{*}=0.02 R_{\odot}, R_{\mathrm{disk}}=20 R_{*}, i=30^{\circ}$; for the ionized gas $T_{e}=15000 \mathrm{~K}, n_{e}=1.0 \cdot 10^{8} \mathrm{~cm}^{-3}$ and for the cold component $\mathrm{Sp}=\mathrm{M} 4 \mathrm{III}, T_{\text {eff }}=3500 \mathrm{~K}, R=141 R_{\odot}$. Explanation of different types of lines see in the text.

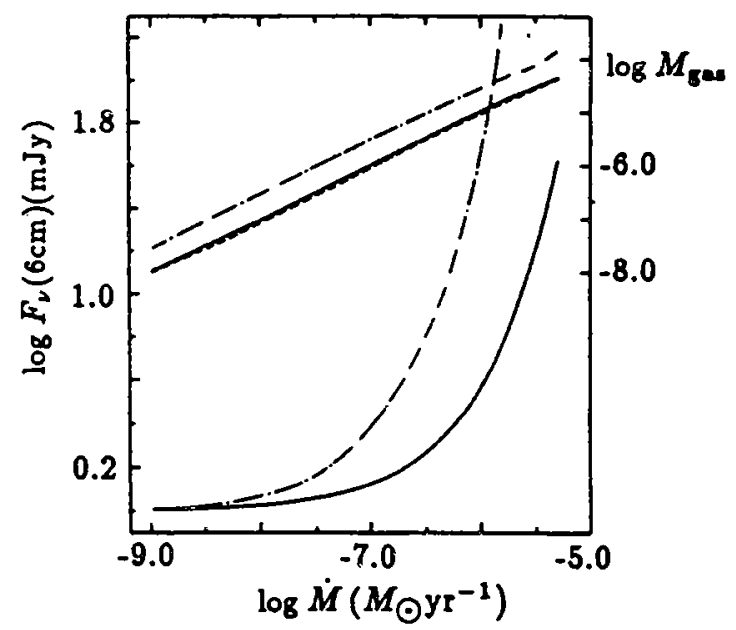

Fig. 15. Dependence of radio flux at $4.9 \mathrm{GHz}(6 \mathrm{~cm})$ and mass of the ionized gas on the accretion rate. Other parameters of the symbiotic system are as in Fig. 14. 
The quantities computed according to this formula are marked with solid lines in Figs. 14 and 15. If gas pressure is important in the boundary layer, this expression must be modified to

$$
T_{\mathrm{BL}} \approx 4.5 \cdot 10^{4}\left(\frac{\dot{M}}{10^{-5} M_{\odot} \mathrm{yr}^{-1}}\right)^{2 / 8}\left(\frac{M_{*}}{M_{\odot}}\right)^{1 / 3}\left(\frac{R_{*}}{R_{\odot}}\right)^{-7 / 9}
$$

Dashed lines in Figs. 14 and 15 mark the quantities computed by using formula (2) for the boundary layer temperature. Dot-dashed lines mark the quantities corresponding to the boundary layer temperature found by the formula

$T_{\mathrm{BL}} \approx 7.6 \cdot 10^{4}\left(\frac{\dot{M}}{10^{-5} M_{\odot} \mathrm{yr}^{-1}}\right)^{6 / 18}\left(\frac{M_{*}}{M_{\odot}}\right)^{8 / 19}\left(\frac{R_{*}}{R_{\odot}}\right)^{-18 / 19}$

which is valid, if the Thomson scattering dominates other opacity sources in the boundary layer.

In Fig. 16 we demonstrate the relations between the accretion rate and the temperature of the boundary layer computed by all three formulae. There is no substantial difference between the values of the temperature found from formulae (1) and (2), while electron scattering yields quite a different picture. The same is seen in Figs. 14 and 15 for other quantities. There is no direct evidence of domination of electron scattering in the accretion disks of symbiotic stars, and in the computations for real symbiotic stars we usually have used formula (1) as a reasonable compromise.

\subsection{Inclination angle of the disk}

Visibility of the accretion disk depends on the orientation of the orbit of a binary star in the space, characterized by the inclination angle $i$. As expected, the physical quantities characterizing ionized gas almost do not depend on the inclination angle of the disk, and only a small decrease in $U-B$ is seen (Fig. 17). Screening of the accretion disk with the increasing of the angle $i$ is expressively characterized by fractional contributions of the hot component and the ionized gas to the total emergent flux of the symbiotic system. Mutual exchanges in these contributions are well seen in Fig. 17. This 


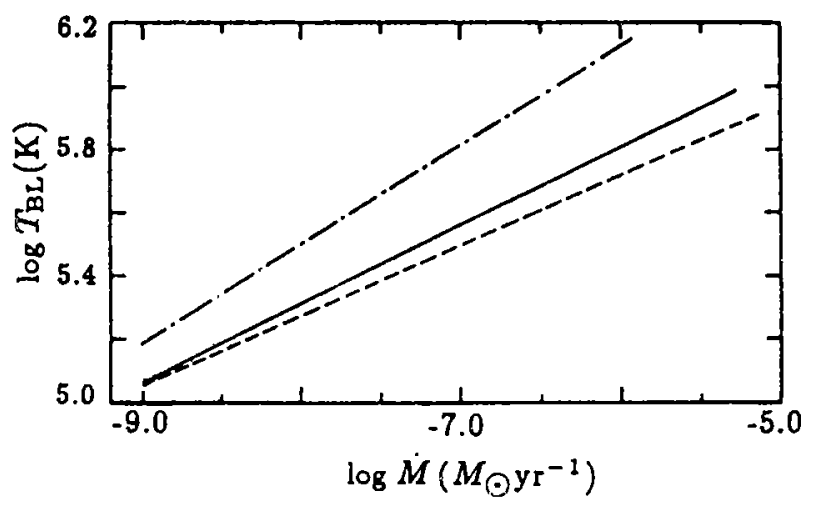

Fig. 16. Temperature of the boundary layer computed by different formulae as a function of the accretion rate. Other parameters of the symbiotic system are as in Fig. 15.

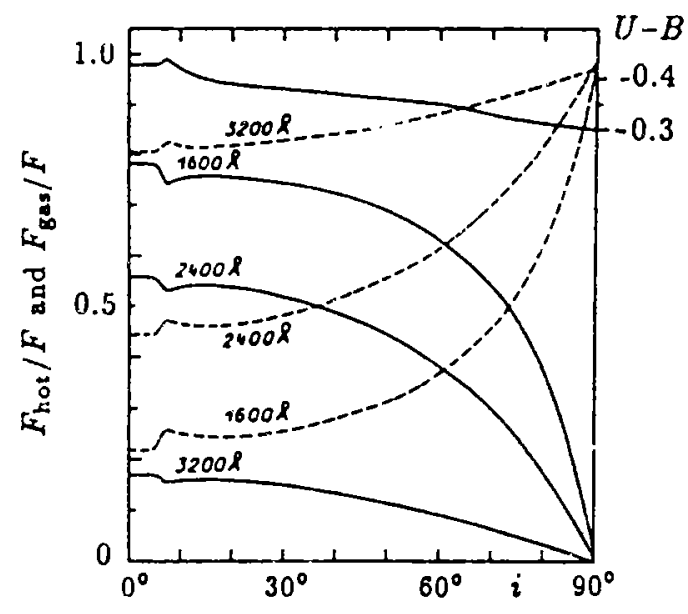

Fig. 17. Fractional contributions of the hot component $F_{\text {hot }} / F$ (solid lines) and of ionized gas $F_{\text {gas }} / F$ (dashed lines) to the total radiation of the symbiotic system as a function of the inclination angle $i$ of the accretion disk. Other parameters of the system are: for the accretor $M=$ $0.90 M_{\odot}, R_{*}=0.012 R_{\odot}, \dot{M}_{\text {acc }}=1.0 \cdot 10^{-7} M_{\odot} \mathrm{yr}^{-1}, R_{\text {disk }}=20 R_{*} ;$ for the ionized gas $T_{e}=10000 \mathrm{~K}, n_{\mathrm{e}}=1.0 \cdot 10^{7} \mathrm{~cm}^{-3}$ and for the cold component $\mathrm{Sp}=\mathrm{M} 4 \mathrm{III}, T_{\mathrm{eff}}=3500 K, R=141 R_{\odot}$. 
dependence appears slightly also in the form of the continuous spectrum.

\section{Cold component of a symbiotic system}

In the optical and infrared spectral regions most of radiation of the symbiotic system comes from the cold component, a red giant. As discussed in our paper (Leedjärv, 1990), the cold component of a symbiotic system is linked to the properties of its gas envelope, and even the luminosities of cold and hot components can be correlated for the symbiotic star phenomenon to be revealed. Therefore, the red giant has prime importance in determining the characteristics of the whole symbiotic system.

Usually, red giants are the best-studied components of symbiotic stars, and when fitting their continuous spectra in a wide wavelength range, we can ascribe definite values to the radius and the effective temperature of the red giant. However, in some cases it may be useful to investigate how the properties of the red giant are linked to the observable characteristics of a symbiotic star. We have taken the values for the radius according to the models by Tsuji (1978) as quantitative parameters for the spectral subclass of the red giant.

If we fix the values of all parameters of a symbiotic system and then vary only the spectral subclass of the red giant, the greatest changes can be expected in the shape of the continuous spectrum in the optical and near-infrared regions. Really, the changes in colour indices $V-R, V-I$ and $V-J$ are very significant (Fig. 18). In Fig. 19 such great changes in far-infrared colour indices can be seen as well. Although in this spectral region the shape of the continuous spectrum of the red giant does not essentially depend on the spectral subclass considered, the absolute values of the emergent flux vary in a wide range and this causes remarkable variations in colour indices. The behaviour of $U-B$ in Fig. 19 can be explained as follows: in the case of an earlier spectral subclass the increase of radius of the red giant increases also its luminosity in the $B$ bandpass and this leads to the reddening of $U-B$ which reaches its maximum value at the spectral subclass M4. At subclasses M5 and M6, in spite of an increase in overall luminosity of the red giant, its luminosity in the $B$ bandpass decreases, which leads to a decrease in the value of $U-B$. 


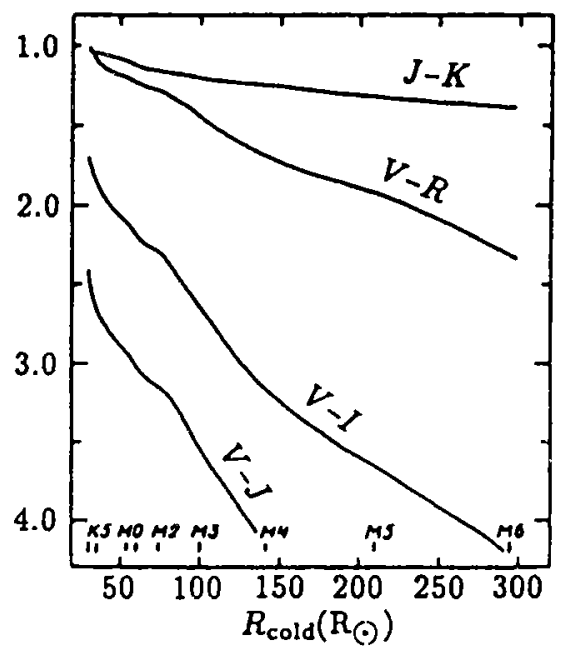

Fig. 18. Dependence of some colour indices on the radius of the cold component. Other parameters of the symbiotic system are: for the hot component (blackbody) $T_{\text {hot }}=70000 \mathrm{~K}, R_{\text {hot }}=0.10 R_{\odot}$ and for the ionized gas $T_{e}=15000 \mathrm{~K}, n_{e}=5.0 \cdot 10^{7} \mathrm{~cm}^{-3}$.

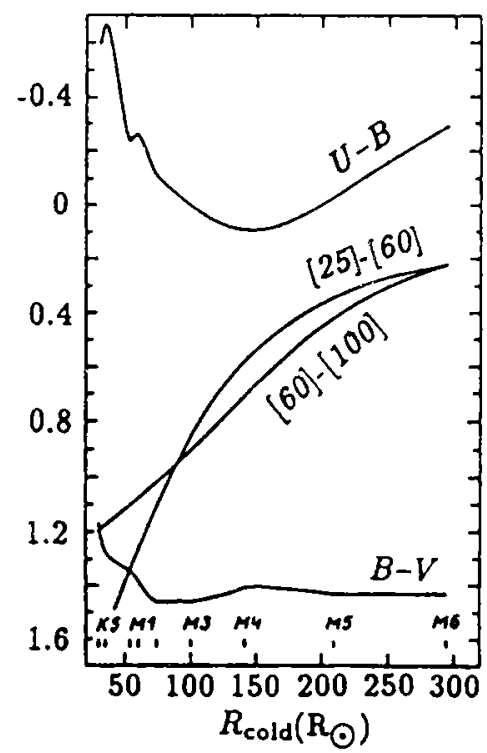

Fig. 19. Dependence of some colour indices on the radius of the cold component. Other parameters of the system are as in Fig. 18. 


\section{Conclusions}

(1) For symbiotic star models with a blackbody-type hot component both the effective temperature and the radius of the blackbody affect remarkably the shape of the continuous spectrum. The amount of radiating ionized gas depends on the hot component luminosity, i.e. on the number of ionizing blackbody photons.

(2) Physical parameters of the ionized gas envelope, electron density and electron temperature do not affect remarkably the continuous spectrum.

(3) If any dust envelope can be found in a symbiotic system, its radius and temperature affect moderately colour indices in both the near- and far-infrared regions.

(4) In the case of an accretion disk as the hot component, the inclination angle of the disk and the mass of the accreting star do not essentially affect the shape of the continuous spectrum, while the radius of the accreting star and the gas accretion rate are very significant parameters, which specify the number of ionizing photons and, consequently, the emergent flux.

(5) The higher the ratio of the luminosities $L_{\text {hot }} / L_{\text {cold }}$, the farther off is the position of symbiotic stars from the sequence of normal $M$-giants in the $U-B, B-V$ diagram. There is a possibility that under certain conditions we can distinguish in this diagram the symbiotic stars with a blackbody-type hot component and with an accretion disk.

(6) In the far-infrared region $(12-100 \mu \mathrm{m})$ two-colour diagrams exhibit a clear distinction between S- and D-type symbiotic stars. Some symbiotic stars hitherto found to be S-type may contain some amount of cold dust $(T \sim 100 \mathrm{~K})$.

Acknowledgments. The author thanks Dr. I. Pustylnik for valuable discussions, T. Haud for linguistic help and E. Mark and T. Kuusk for their help in preparing the Figures.

\section{References}

Anandarao, B. G. and Pottasch, S. R. 1986, A\&A, 162, 167.

Anandarao, B. G., Taylor, A. R. and Pottasch, S. R. 1988, A\&A, 203, 361. Beichman, C. A., Neugebauer, G., Habing, H. J., Clegg, P. E. and Chester, T.J. 1985, IRAS Explanatory Supplement (Washington, US GPO). Johnson, H. L. 1965, ApJ, 141, 923. 
Kaler, J. B. 1976, ApJ, 210, 843.

Kenyon, S. J., Fernandez-Castro, T. and Stencel, R. E. 1988, AJ, 95, 1817. Kenyon, S. J. and Webbink, R. F. 1984, ApJ, 279, 252.

Leedjärv, L. 1988, Est. Acad. Sci. Prepr. A-7, Tallinn.

Leedjärv, L. 1989, Astrofizika, 31, 309.

Leedjärv, L. 1990, Astrofizika, 32, 15.

Luud, L. and Tuvikene, T. 1987, Astrofizika, 26, 457.

Lynden-Bell, D. and Pringle, J. E. 1974, MNRAS, 168, 603.

Pringle, J. E. 1977, MNRAS, 178, 195.

Seaquist, E. R. and Taylor, A. R. 1990, ApJ, 349, 313.

Shakura, S. and Sunyaev, R. A. 1973, A\&A, 24, 337.

Straiżys, V. 1977, Multicolour Stellar Photometry (Vilnius, Mokslas Publishers).

Straižys, V. and Sviderskiene, Z. 1972, Bull. Vilnius Obs., No.35, 3.

Sviderskiene, Z. 1974, Bull. Vilnius Obs., No.40, 26.

Tsuji, T. 1978, A\&A, 62, 29. 
